\title{
Stool methanogens in intestine mammal species.
}

2

Guindo C.O.1,2, Davoust B², Drancourt $\mathrm{M}^{1,2}$, Grine $\mathrm{G}^{2,3 *}$.

4

1. IHU Méditerranée Infection, Marseille, France.

2. Aix-Marseille Université, IRD, MEPHI, IHU Méditerranée Infection, Marseille, France.

3. Aix-Marseille-Université, Faculty of Odontology, Marseille, France.

9

${ }^{*}$ Corresponding author: Ghiles GRINE, PhD

11 IHU Méditerranée Infection, UMR MEPHI

12 19-21, Bd Jean Moulin 13005 Marseille, France. Tel: +33 (0)4 137324 01, fax: + 33

13

(0) 13732402 .

14

Email: grineghiles@univ-amu.fr. 


\section{INTRODUCTION}

Methanogens are archaea characterized by their unique capability in producing methane from by-products of bacterial anaerobe fermentations; being members of anaerobe microbiota of the digestive tract microbiota of several mammals (1). Accordingly, methanogens gained interest in the clinical microbiology over the last years after methanogens have been detected by PCR-based methods and cultured from the gut microbiota $(2,3)$; and their translocation in milk and urines has been further observed (4). Moreover, methanogens have been associated with dysbiosis such as in the case of vaginosis (5), urinary tract infections (6) and anaerobe abscesses of the oral cavity in the case of periodontitis and periimplantitis $(7,8)$, in the case of refractory sinusitis $(9)$, brain $(10,11)$ and muscle $(12)$. Recently, we observed blood-borne methanogens associated with endocarditis (13). In all these situations, anaerobe bacteria were associated in the methanogen disease process and this observation was probably reflecting methanogen specificities including the absolute oxygen intolerance and the necessity of a hydrogen source to produce methane $(14,15)$.

Currently, 16 different methanogens have been cultured from digestive tract microbiota of mammals (16-19) and PCR-based methods of detecting speciesspecific sequences traced an additional 04 species (18) (Table 1).

The sources, modes of acquisition and dynamics of digestive tract methanogens remain poorly investigated. We previously reported that the one-day newborns exhibited culturable Methanobrevibacter smithii (M. smithii) in the gastric fluid (20), suggesting a perinatal source of acquisition. Accordingly, we reported that mother milk did contain culturable M. smithii and culturable Methanobrevibacter oralis (M. oralis) (4). Yet, it is unclear whether these one-day methanogens do persist 
along with the digestive tract of the newborns or whether this is just one of several waves of acquisition of methanogens along the first months of the life (20-24). Therefore, the search for methanogens sources other than the mother milk is of interest.

Certain mammals (cow, sheep, donkey, horse, cat, pig, rabbit, rat, rhinoceros, baboon, monkey, and hippopotamus); birds (goose, turkey, and chicken) and insects (termites) are acknowledged to harbor digestive tract methanogens and $M$. smithii in particular has already been detected from bovine and also from Wistar rats $(18,19,25-40)$. In this study, we aimed to expand the spectrum of animals which could be sources of methanogens for human, by exploring methanogen carriage in animals in contact with the general population or with some restricted populations; comparing the repertoire of animal methanogens with the one of human methanogens in order to question methanogens as zoonotic microorganisms.

MATERIALS AND METHODS

Feces samples. Feces are legally regarded as garbage in FRANCE and their collection does not require any institutional approval. In the absence of any legal obligation, we asked the owners' approval for feces collections and proceeding.

After the obtention of the verbal consent from animals owners, feces samples have been collected from nine different animal species including cat, dog, horse, sheep, rabbit, cow, pigs, goat, and donkey; from animals living in metropolitan France, more precisely in the Marseille metropolitan area, Southeastern France (Table 2). Dogs and cats were fed dry industrial dry kibble feed; horses were fed hay + straw + pellets; sheep and goats were fed pasture (grass) and dry supplementary feed; 
rabbits were fed dehydrated alfalfa + hay + pellets (other vegetables, cereals,

mineral salts and vitamins); cows were fed hay + straw + pasture (grass) and whole

plant maize silage; pigs were fed straw + dry pelleted feed (formula consisting mainly of maize, wheat, oats, peas, soybeans, cereals, oilseeds and minerals) and donkeys were fed grass and hay. Feces samples have been stored at $+4^{\circ} \mathrm{C}$ for five weeks before being process for DNA extraction as reported below.

DNA extraction and PCR assays. DNA extraction was performed by mixing $0.2 \mathrm{~g}$ of each feces sample with $500 \mu \mathrm{L}$ of $\mathrm{G} 2$ buffer (QIAGEN, Hilden, Germany) in an Eppendorf tube (Fisher Scientific, Illkirch, France). Then, $0.3 \mathrm{~g}$ of acid-washed beads $\leq 106 \mu \mathrm{m}$ (Sigma-Aldrich, Saint-Quentin Fallavier, France) were added in each tube and shake in a FastPrep BIO 101 device (MP Biomedicals, Illkirch, France) for 45 seconds for mechanical lysis before 10 -minute incubation at $100^{\circ} \mathrm{C}$. A $180 \mu \mathrm{L}$ volume of the mixture was then incubated with $20 \mu \mathrm{L}$ of proteinase K (QIAGEN) at $56^{\circ} \mathrm{C}$ overnight before a second mechanical lysis was performed. Total DNA was finally extracted with the EZ1 Advanced XL extraction kit (QIAGEN) and $200 \mu \mathrm{L}$ eluted volume. Sterile phosphate-buffered saline (PBS) was used as a negative control in each DNA extraction run. Extracted DNA was incorporated into real-time PCR performed using Metha_16S_2_MBF: 5'-CGAACCGGATTAGATACCCG -3' and Metha_16S_2_MBR: 5'- CCCGCCAATTCCTTTAAGTT-3' primers (Eurogentec, Angers, France) and FAM_Metha_16S_2_MBP 6FAMCCTGGGAAGTACGGTCGCAAG probe targeting the 16S DNA gene of methanogens, designed in our laboratory (Eurogentec). PCR amplification was done in a $20 \mu \mathrm{L}$ volume including $15 \mu \mathrm{L}$ of mix and $5 \mu \mathrm{L}$ of extracted DNA. Five $\mu \mathrm{L}$ of ultrapure water (Fisher Scientific) were used instead of DNA in the negative controls. The amplification reaction was performed in a CFX96 thermocycler (BioRad, Marnes-la- 
90 Coquette, France) incorporating a protocol with a cycle of $50^{\circ} \mathrm{C}$ for 2 -minute, followed by 39 cycles of $95^{\circ} \mathrm{C}$ for 5 -minute, $95^{\circ} \mathrm{C}$ for 5 seconds and finally $60^{\circ} \mathrm{C}$ for

YCCGGCGTTGAMTCCAATT-3) genes was performed as previously described [8,

BigDye Terminator, version 1.1, cycle sequencing kit DNA according to the sequences were assembled using Chromas Pro software, version 1.7 (Technelysium

Pty Ltd., Tewantin, Australia) and compared with sequences available in the

GenBank database using the online NCBI BLAST program

(http://blast.ncbi.nlm.nih.gov.gate1.inist.fr/Blast.cgi). We considered the sequences as belonging to the same species if the percentage of identity was $>98.7 \%$; as different species if between 95-98.7\% and different genera if this threshold was < $95 \%$ with respect to the first hit obtained by BLAST (41).

\section{Multispacer sequence typing.}

We carried-out a multispacer sequence typing (MST) technique on all fecal specimens positive by PCR-sequencing as previously described in our laboratory $(20,42)$. PCRs were realized in a 2720 Thermal Cycler (Applied Biosystems, Foster City, USA) and followed all the steps described for standard PCR used for the molecular analysis of fecal specimens. Negative controls consisting of PCR mixture

111 without DNA template were included in each PCR run. All PCR products were sequenced in both directions using the same primers as used for PCRs in a 2720 Thermal Cycler (Applied Biosystems) with an initial 1-minute denaturation step at $96^{\circ} \mathrm{C}$, followed by 25 cycles denaturation for 10 seconds each at $96^{\circ} \mathrm{C}$, a 20 seconds 
annealing step at $50^{\circ} \mathrm{C}$ and a 4 -minute extension step at $60^{\circ} \mathrm{C}$. Sequencing products were purified using the MultiScreen 96-well plates Millipore (Merck, Molsheim,

117 France), containing $5 \%$ of Sephadex G-50 (Sigma-Aldrich), and sequences were 118 analyzed on an ABI PRISM 31309 Genetic Analyzer (Applied Biosystem, Foster City, 119 USA) and edited using the ChromasPro software (version 1.42; Technelysium Pty Ltd). For each intergenic spacer, a spacer type (ST) was defined as a sequence exhibiting unique genetic polymorphism (SNPs and indels). MST genotypes were defined as a unique combination of the four spacer sequences $(20,42)$.

Phylogenetic analyses.

Sequences were edited using ChromasPro software (ChromasPro 1.7, evolutionary analyses were conducted in MEGA7 as previously described (43).

\section{Statistical analyses.}

We used R software for data analysis (https://www.r-project.org/). The Chi 2 test was used to compare the prevalence between the different animal species with a threshold $\alpha=0.05$.

\section{RESULTS}

In this study, a total of 407 fecal specimens collected from nine different mammalian species were investigated by RT-PCR for the presence of methanogens (Table 2).

135 Incorporating the 16S rRNA archaeal gene PCR primers newly designed in our 136 laboratory into RT-PCR, we detected the presence of methanogen DNA in all 
mammals here investigated and none of the negative controls. $100.0 \%$ of cat feces specimens were positive with Ct values of $33.51 \pm 1.28 ; 78.8 \%$ of dog feces specimens were positive with Ct values of $27.71 \pm 0.94 ; 84.4 \%$ of horse feces specimens were positive with Ct values of $25 \pm 2.95 ; 96.6 \%$ of sheep feces specimens were positive with Ct values of $27.19 \pm 3.11 ; 100 \%$ of rabbit feces specimens were positive with Ct values of $27.1 \pm 1.36 ; 100 \%$ of cow feces specimens were positive with Ct values of $24.11 \pm 1.94 ; 100 \%$ of pig feces specimens were positive with Ct values of $22.15 \pm 2.75 ; 80 \%$ of goat feces specimens were positive with Ct values of $19.18 \pm 2.46$ and $100 \%$ of donkey feces specimens were positive with Ct values of $18.82 \pm 1.44$ (Table 3 and Table 4).

147 Prevalence as a function of RT-PCR differs significantly ( $p$-value<0.05) between animal species (Table 4).

149 Sequencing the PCR products was used to precise the identification of methanogens at the genus and species levels, in each sample. In cats, 50/105 successfully sequenced samples yielded 20 Methanocorpusculum aggregans (M. aggregans), 13 Methanocorpusculum labreanum (M. labreanum), 09 Methanobrevibacter millerae (M. millerae), 04 M. smithii, 02 Methanobrevibacter thaueri (M. thaueri), and 02 Methanobrevibacter olleyae (M. olleyae). In dogs, 30/52 successfully sequenced samples yielded 13 M. labreanum, 06 M. smithii, 05 M. aggregans, 03 M. thaueri, 02 M. millerae, and 01 M. olleyae. In horses, 24/89 successfully sequenced samples

157 yielded 11 M. aggregans, 10 M. olleyae, 01 M. smithii, 01 M. millerae, and 01 M. 158 labreanum. In sheep, 28/29 successfully sequenced samples yielded $23 \mathrm{M}$. labreanum, 03 M. millerae, 01 M. smithii, and 01 M. aggregans. In rabbits, 2/2 successfully sequenced samples yielded 02 M. thaueri. In cows, $44 / 57$ successfully sequenced samples yielded $22 \mathrm{M}$. aggregans, $11 \mathrm{M}$. millerae, $06 \mathrm{M}$. labreanum and 
05 M. thaueri. In pigs, 25/64 successfully sequenced samples yielded 12 M. smithii,

09 M. millerae, 03 Methanomassiliicoccus luminiyensis (M. luminiyensis) and $01 \mathrm{M}$.

olleyae. In goats, 4/5 successfully sequenced samples yielded 03 M. labreanum and

01 M. aggregans. Finally, in donkeys, 4/4 successfully sequenced samples yielded

04 M. aggregans (Fig 1). So, we had 55/105 sequencing failures in cats; 11/41 in

dogs; $51 / 75$ in horses; $13 / 57$ in cats and 39/64 in pigs. There were no sequencing

failures in sheep, rats, goats, and donkeys (Table 5). Prevalence as a function of

PCR-sequencing differs significantly $(p$-value $<0.05)$ between animal species (Table

$6)$.

We obtained a total of seven different species of methanogens in our study, of which

172 three (M. smithii, M. millerae and M. Iuminyensis) are known to be part of the methanogens present in the human digestive tract (Fig 2 and Fig 3). The remaining four (M. thaueri, M. olleyae, M. labreanum and $M$. aggregans) are not known to date in humans. However, we did not find in our study the other ten species of methanogens present in the human digestive tract including Methanobrevibacter arboriphilicus, M. oralis, Methanosphaera stadtmanae (M. stadtmanae), Candidatus Methanomethylophilus alvus (Ca. Methanomethylophilus alvus), Candidatus Methanomassiliicoccus intestinalis (Ca. Methanomassiliicoccus intestinalis), Methanoculleus chikugoensis (M. chikugoensis), Methanobacterium congolense (M. congolense), Methanoculleus bourgensis (M. bourgensis), Candidatus

182 Nitrososphaera evergladensis (Ca. Nitrososphaera evergladensis) and 183 Methanosarcinia mazei (M. mazei) (Fig 2 and Fig 3).

184 Among the 211 sequences obtained, $153(72.51 \%)$ of them have an identity 
$98.7 \%$ and $15(7.10 \%)$ have an identity percentage lower than 95\% (Table 7 ). The

187

188

189

190

191

192

193

194

195

196

197

198

199

200

201

202

203

204

205

206

207

phylogenetic trees of sequences obtained with a percentage identity lower than

$98.7 \%$ and sequences with a percentage identity lower than $95 \%$ indicated new

species and new genera respectively (Fig 4 and Supplementary Figures). We

obtained 24 M. smithii by PCR-sequencing including 12/24 (50\%) in pigs, 6/24 (25\%)

in dogs, $4 / 24(16.66 \%)$ in cats, and $1 / 24(4.16 \%)$ in both sheep and horses (Table 8 ).

Genotyping the $24 M$. smithii revealed five different genotypes. Genotype 1 was

found in 8/24 (33.33\%); genotype 2 in 10/24 (41.66\%); genotype 3 in 4/24 (16.66\%);

genotypes 4 and 5 in 1/24 (4.16\%) each (Table 8).

\section{DISCUSSION}

It is known and published that methanogens colonize the gastrointestinal tract of certain mammals, particularly herbivorous ones [35]. Most methanogens identified in mammals belong to the phylum Euryarchaeota, with a high percentage of the species M. smithii [36], a species being the most prevalent one in humans (44). Our report is the largest one showing the presence of methanogens in nine mammals in the same study. Our results confirmed the published data on the presence of methanogens in the digestive tract of cats, dogs, horses, cows, sheep, rabbits, goats, pigs, and donkeys $(18,19,25-31,33-35)$. In addition, all methanogens found in this study belong to the phylum Euryarchaeota, which is in accordance with the results obtained in studies conducted on the human digestive tract $(45,46)$. Our results give an insight on the concentration of methanogens present in the intestinal microbiota of each animal species analyzed and on the prevalence of methanogens in domestic animals by humans. 
The results of the analysis of the 16S RNA sequences obtained from our samples show that there is a real diversity of methanogenic archaea genera

(Methanosphaera, Methanocorpusculum, Methanocalculus, Methanoculleus,

Methanogenium, Methanoplanus, Methanolacinia, Methanobacterium,

Methanomicrobium, Methanomassiliicoccus and Methanobrevibacter) in the digestive tract of animals (cats, dogs, horses, sheep, cows, rabbits, goats, pigs and donkeys) as in humans $(45,46)$. All sequences with a percentage lower than $98.7 \%$ have been deposited in the GenBank database (accession no MT587812 to MT587864) and EBI database (accession no MT793590; MT819603; MT822292; MT822293; and MT822482).

219 This study demonstrated for the first time the presence of the genus Methanomassiliicoccus in animals, specifically $M$. luminiyensis in pigs. This methanogen was until now known only in humans (47). This could be explained by the fact that the pig is an omnivore which means that its diet is very close to that of humans compared to other animals. However, the only time this species was isolated and cultured in humans was in a healthy 86-year-old Caucasian man who most probably consumed pig (47). This suggested a probable link between consumption of pig meat and/or contact with pig and the presence of $M$. luminiyensis in humans. In addition, $50 \%$ of $M$. smithii in our study were found in pigs, indicating that M. smithii was the most prevalent methanogen in the digestive tract of pigs, consistent with work carried out in humans where the high prevalence of $M$. smithii in the digestive tract has been demonstrated $(44,46)$.

These results are fairly representative of the methanogen community present in the digestive tract of certain animals domesticated by humans and other future studies 
must be done to try to cultivate methanogens here detected by molecular biology to

234 better understand the dynamics of methanogens in animals and also the likely

235 acquisition of methanogens in humans through direct contact with these animals or

236 through consumption of the meat and/or milk of certain animals, in particular cows,

237 since a recent study has demonstrated an association between the acquisition of $M$.

238 smithii in children and the consumption of dairy products (48). 
bioRxiv preprint doi: https://doi.org/10.1101/2020.08.24.264788; this version posted August 24, 2020. The copyright holder for this preprint (which was not certified by peer review) is the author/funder, who has granted bioRxiv a license to display the preprint in perpetuity. It is made available under aCC-BY 4.0 International license.

\section{ACKNOWLEDGEMENTS}

241 This work was supported by a grant of GDR Archaea, Centre National de la

242 Recherche Scientifique, Paris, France. 


\section{REFERENCES}

1. Michelland RJ, Monteils V, Combes S, Cauquil L, Gidenne T, Fortun-Lamothe L. Comparison of the archaeal community in the fermentative compartment and faeces of the cow and the rabbit. Anaerobe. août 2010;16(4):396-401.

2. Nkamga VD, Henrissat B, Drancourt M. Archaea: Essential inhabitants of the human digestive microbiota. Human Microbiome Journal. mars 2017;3:1-8.

3. Guindo CO, Drancourt M, Grine G. Digestive tract methanodrome: Physiological roles of human microbiota-associated methanogens. Microbial Pathogenesis. juill 2020;104425.

4. Togo AH, Grine G, Khelaifia S, des Robert C, Brevaut V, Caputo A, et al. Culture of Methanogenic Archaea from Human Colostrum and Milk. Sci Rep. déc 2019;9(1):18653.

5. Grine G, Drouet H, Fenollar F, Bretelle F, Raoult D, Drancourt M. Detection of Methanobrevibacter smithii in vaginal samples collected from women diagnosed with bacterial vaginosis. Eur J Clin Microbiol Infect Dis. sept 2019;38(9):1643-9.

6. Grine G, Lotte R, Chirio D, Chevalier A, Raoult D, Drancourt M, et al. Co-culture of Methanobrevibacter smithii with enterobacteria during urinary infection. EBioMedicine. mai 2019;43:333-7.

7. Faveri M, Gonçalves LFH, Feres M, Figueiredo LC, Gouveia LA, Shibli JA, et al. Prevalence and microbiological diversity of Archaea in peri-implantitis subjects by $16 \mathrm{~S}$ ribosomal RNA clonal analysis: Archaea in peri-implantitis subjects. Journal of Periodontal Research. juin 2011;46(3):338-44. 
8. Nguyen-Hieu T, Khelaifia S, Aboudharam G, Drancourt M. Methanogenic

9. Sogodogo E, Fellag M, Loukil A, Nkamga VD, Michel J, Dessi P, et al. Nine Entity. Front Public Health. 4 mars 2019;7:38.

10. Drancourt M, Nkamga VD, Lakhe NA, Régis J-M, Dufour H, Fournier P-E, et al. Evidence of Archaeal Methanogens in Brain Abscess. Clinical Infectious Diseases. 1 juill 2017;65(1):1-5.

11. Nkamga VD, Lotte R, Chirio D, Lonjon M, Roger P-M, Drancourt M, et al. Methanobrevibacter oralis detected along with Aggregatibacter actinomycetemcomitans in a series of community-acquired brain abscesses. Clinical Microbiology and Infection. févr 2018;24(2):207-8.

12. Nkamga VD, Lotte R, Roger P-M, Drancourt M, Ruimy R. Methanobrevibacter smithii and Bacteroides thetaiotaomicron cultivated from a chronic paravertebral muscle abscess. Clinical Microbiology and Infection. déc 2016;22(12):1008-9.

13. Drancourt M, Djemai K, Gouriet F, Grine G, Loukil A, Bedotto M, et al. Methanobrevibacter smithii archaemia in febrile patients with bacteremia, including those with endocarditis. Clinical Infectious Diseases. 15 juill 2020;ciaa998.

14. Sogodogo E, Drancourt M, Grine G. Methanogens as emerging pathogens in anaerobic abscesses. Eur J Clin Microbiol Infect Dis. mai 2019;38(5):811-8. 
15. Guindo CO, Terrer E, Chabrière E, Aboudharam G, Drancourt M, Grine G. Culture of salivary methanogens assisted by chemically produced hydrogen. Anaerobe. févr 2020;61:102128.

16. Miller TL. Description of Methanobrevibacter gottschalkii sp. nov., Methanobrevibacter thaueri sp. nov., Methanobrevibacter woesei sp. nov. and Methanobrevibacter wolinii sp. nov. INTERNATIONAL JOURNAL OF SYSTEMATIC AND EVOLUTIONARY MICROBIOLOGY. 1 mai 2002;52(3):819-22.

17. Pol A, Demeyer DI. Fermentation of methanol in the sheep rumen. Applied and 296 Environmental Microbiology. 1988;54(3):832-4.

18. Janssen PH, Kirs M. Structure of the Archaeal Community of the Rumen. Appl Environ Microbiol. 15 juin 2008;74(12):3619-25.

19. Jarvis GN, Strömpl C, Burgess DM, Skillman LC, Moore ERB, Joblin KN. Isolation and Identification of Ruminal Methanogens from Grazing Cattle. Current Microbiology. 1 mai 2000;40(5):327-32.

20. Grine G, Boualam MA, Drancourt M. Methanobrevibacter smithii, a methanogen consistently colonising the newborn stomach. Eur J Clin Microbiol Infect Dis. déc 2017;36(12):2449-55.

21. Mihajlovski A, Doré J, Levenez $F$, Alric $M$, Brugère J-F. Molecular evaluation of the human gut methanogenic archaeal microbiota reveals an age-associated increase of the diversity. Environmental Microbiology Reports. avr 2010;2(2):272-80. 
22. Danielsson R, Schnürer A, Arthurson V, Bertilsson J. Methanogenic Population and $\mathrm{CH} 4$ Production in Swedish Dairy Cows Fed Different Levels of Forage. Appl Environ Microbiol. 1 sept 2012;78(17):6172-9.

23. Odamaki T, Kato K, Sugahara H, Hashikura N, Takahashi S, Xiao J, et al. Agerelated changes in gut microbiota composition from newborn to centenarian: a cross-sectional study. BMC Microbiology. 25 mai 2016;16(1):90.

24. Palmer C, Bik EM, DiGiulio DB, Relman DA, Brown PO. Development of the Human Infant Intestinal Microbiota. Ruan Y, éditeur. PLoS Biol. 26 juin 2007;5(7):e177.

25. Miller TL, Wolin MJ. Methanogens in human and animal intestinal Tracts. Systematic and Applied Microbiology. mai 1986;7(2-3):223-9.

26. Miller TL, Wolin MJ, Zhao HX, Bryant MP. Characteristics of methanogens isolated from bovine rumen. Applied and Environmental Microbiology. 1986;51(1):201-2.

27. Skillman LC, Evans PN, Strömpl C, Joblin KN. 16S rDNA directed PCR primers and detection of methanogens in the bovine rumen. Letters in Applied Microbiology. 2006;42(3):222-8.

28. Zhang Y, Zamudio Cañas EM, Zhu Z, Linville JL, Chen S, He Q. Robustness of archaeal populations in anaerobic co-digestion of dairy and poultry wastes. Bioresource Technology. 1 janv 2011;102(2):779-85. 
29. Luo Y, Su Y, Wright A-DG, Zhang L, Smidt H, Zhu W. Lean Breed Landrace Pigs Harbor Fecal Methanogens at Higher Diversity and Density than Obese Breed Erhualian Pigs. Archaea. 2012;2012:1-9.

30. Hernandez-Sanabria E, Goonewardene LA, Wang Z, Zhou M, Moore SS, Guan LL. Influence of Sire Breed on the Interplay among Rumen Microbial Populations Inhabiting the Rumen Liquid of the Progeny in Beef Cattle. Zhou Z, éditeur. PLoS ONE. 8 mars 2013;8(3):e58461.

31. Rea S, Bowman JP, Popovski S, Pimm C, Wright A-DG. Methanobrevibacter millerae sp. nov. and Methanobrevibacter olleyae sp. nov., methanogens from the ovine and bovine rumen that can utilize formate for growth. International Journal of Systematic and Evolutionary Microbiology. 1 mars 2007;57(3):450-6.

32. Murru F, Fliegerova K, Mura E, Mrázek J, Kopečný J, Moniello G. A comparison of methanogens of different regions of the equine hindgut. Anaerobe. déc 2018;54:104-10.

33. Wright A-DG, Williams AJ, Winder B, Christophersen CT, Rodgers SL, Smith KD. Molecular Diversity of Rumen Methanogens from Sheep in Western Australia. Appl Environ Microbiol. mars 2004;70(3):1263-70.

34. Lee J-H, Kumar S, Lee G-H, Chang D-H, Rhee M-S, Yoon M-H, et al. Methanobrevibacter boviskoreani sp. nov., isolated from the rumen of Korean native cattle. INTERNATIONAL JOURNAL OF SYSTEMATIC AND EVOLUTIONARY MICROBIOLOGY. 1 nov 2013;63(Pt 11):4196-201. 
35. Tun HM, Brar MS, Khin N, Jun L, Hui RK-H, Dowd SE, et al. Gene-centric metagenomics analysis of feline intestinal microbiome using 454 junior pyrosequencing. Journal of Microbiological Methods. mars 2012;88(3):369-76.

36. Beauchemin KA, Ungerfeld EM, Eckard RJ, Wang M. Review: Fifty years of research on rumen methanogenesis: lessons learned and future challenges for mitigation. Animal. mars 2020;14(S1):s2-16.

37. Qin $\mathrm{H}$, Lang $\mathrm{H}$, Yang $\mathrm{H}$. Characterization of the methanogen community in a household anaerobic digester fed with swine manure in China. Appl Microbiol Biotechnol. sept 2013;97(18):8163-71.

38. Saengkerdsub S, Ricke SC. Ecology and characteristics of methanogenic archaea in animals and humans. Critical Reviews in Microbiology. mai 2014;40(2):97-116.

39. Whitford MF, Teather RM, Forster RJ. Phylogenetic analysis of methanogens from the bovine rumen. BMC Microbiology. 2001;5.

40. Mukumba P, Department of Physics, University of Fort Hare, Alice, South Africa, Makaka G, Department of Physics, University of Fort Hare, Alice, South Africa, Mamphweli S, Fort Hare Institute of Technology, University of Fort Hare, Alice, South Africa. Anaerobic digestion of donkey dung for biogas production. S Afr J Sci [Internet]. 27 juill 2016 [cité 29 avr 2020];Volume 112(Number 7/8). Disponible sur: http://sajs.co.za/article/view/3574

41. Stackebrandt E, Ebers J. Taxonomic parameters revisited: tarnished gold standards. MICROBIOLOGY TODAY. 2006;33(4):152-5. 
42. Nkamga VD, Huynh HTT, Aboudharam G, Ruimy R, Drancourt M. Diversity of Human-Associated Methanobrevibacter smithii Isolates Revealed by Multispacer Sequence Typing. Curr Microbiol. juin 2015;70(6):810-5.

43. Kumar S, Stecher G, Tamura K. MEGA7: Molecular Evolutionary Genetics Analysis Version 7.0 for Bigger Datasets. Mol Biol Evol. juill 2016;33(7):1870-4.

44. Dridi B, Henry M, El Khéchine A, Raoult D, Drancourt M. High Prevalence of Methanobrevibacter smithii and Methanosphaera stadtmanae Detected in the Human Gut Using an Improved DNA Detection Protocol. Dobrindt U, éditeur. PLoS ONE. 17 sept 2009;4(9):e7063.

45. Dridi B, Raoult D, Drancourt M. Archaea as emerging organisms in complex human microbiomes. Anaerobe. avr 2011;17(2):56-63.

46. Nkamga VD, Henrissat B, Drancourt M. Archaea: Essential inhabitants of the human digestive microbiota. Human Microbiome Journal. mars 2017;3:1-8.

47. Dridi B, Fardeau M-L, Ollivier B, Raoult D, Drancourt M. Methanomassiliicoccus luminyensis gen. nov., sp. nov., a methanogenic archaeon isolated from human faeces. INTERNATIONAL JOURNAL OF SYSTEMATIC AND EVOLUTIONARY MICROBIOLOGY. 1 août 2012;62(Pt 8):1902-7.

48. van de Pol JAA, van Best N, Mbakwa CA, Thijs C, Savelkoul PH, Arts ICW, et al. Gut Colonization by Methanogenic Archaea Is Associated with Organic Dairy Consumption in Children. Front Microbiol [Internet]. 10 mars 2017 [cité 2 août 2020];8. Disponible sur: http://journal.frontiersin.org/article/10.3389/fmicb.2017.00355/full 
394 Table 1. Methanogens found in digestive tract microbiota of mammals.

395

\begin{tabular}{ll}
\multicolumn{1}{c}{ Culture } & \multicolumn{1}{c}{$\begin{array}{c}\text { Additional species detected by PCR- based } \\
\text { methods }\end{array}$} \\
\hline $\begin{array}{l}\text { Methanosarcina sp., Methanobacterium formicicum, } \\
\text { Methanomicrobium mobile, Methanosarcina barkeri, } \\
\text { Methanobacterium bryantii, Methanobrevibacter, } \\
\text { ruminantium, Methanobrevibacter millerae, }\end{array}$ & $\begin{array}{l}\text { M. smithii, Methanimicrococcus spp., } \\
\text { Methanosphaera spp., Methanobacterium spp. }\end{array}$ \\
Methanobrevibacter olleyae, Methanoculleus & \\
olentangyi, Methanobrevibacter woesei, \\
Methanobrevibacter gottschalkii, Methanobrevibacter \\
thaueri, Methanobrevibacter wolinii, \\
Methanobrevibacter cuticularis, Methanobrevibacter \\
curvatus, Methanobrevibacter filiformis
\end{tabular}

397 Table 2. Details of 407 feces samples here investigated for the presence of methanogens.

\begin{tabular}{|c|c|c|}
\hline Origin of samples & Collected number per sample & Collection sites \\
\hline Cat & 105 & Marseille \\
\hline Dog & 52 & Marseille \\
\hline Horse & 89 & Marseille and Carnoux \\
\hline Sheep & 29 & Bourganeuf \\
\hline Rabbit & 2 & Allauch \\
\hline Cow & 57 & Bourganeuf and Allauch \\
\hline Pig & 64 & Avignon \\
\hline Goat & 5 & Allauch \\
\hline Donkey & 4 & Allauch \\
\hline
\end{tabular}


400 Table 3. Mean and standard deviation of the Ct values obtained in positive samples.

\begin{tabular}{lllllllll}
\hline Cat & Dog & Horse & Sheep & Rabbit & Cow & Pig & Goat & Donkey \\
\hline $33.51 \pm 1.28$ & $27.71 \pm 0.94$ & $25 \pm 2.95$ & $27.19 \pm 3.11$ & $27.1 \pm 1.36$ & $24.11 \pm 1.94$ & $22.15 \pm 2.75$ & $19.18 \pm 2.46$ & $18.82 \pm 1.44$ \\
\hline
\end{tabular}

402 Table 4. Comparison of prevalence based on RT-PCR between animal species.

403

\begin{tabular}{lcccc}
\hline Animal species & $\begin{array}{c}\text { Number of } \\
\text { samples analyzed }\end{array}$ & $\begin{array}{c}\text { Number of } \\
\text { positive samples } \\
\text { by RT-PCR }\end{array}$ & Prevalence [IC 95\%] & p-value \\
\hline Cat & 105 & 105 & $100.0[96.5-100.0]$ & \\
\hline Dog & 52 & 41 & $78.8[65.3-88.9]$ & \\
\hline Horse & 89 & 75 & $84.4[75.0-91.1]$ & \multirow{2}{*}{$9.9 \times 10^{-8}$} \\
\hline Sheep & 29 & 28 & $96.6[82.2-99.9]$ & \\
\hline Rabbit & 2 & 2 & $100.0[15.8-100.0]$ & \\
\hline Cow & 57 & 57 & $100.0[93.7-100.0]$ & \\
\hline Pig & 64 & 64 & $80.0[94.4-100.0]$ & \\
\hline Goat & 5 & 4 & $100.0[39.4-99.5]$ & \\
\hline Donkey & 4 & 4 & & \\
\hline
\end{tabular}

404

405 Table 5. Number of sequencing failures observed in RT-PCR positive samples.

406

\begin{tabular}{lcc}
\hline Animal species & Number of positive samples by RT-PCR & Number of sequencing failures \\
\hline Cat & 105 & $55 / 105$ \\
\hline Dog & 41 & $11 / 41$ \\
\hline Horse & 75 & $51 / 75$ \\
\hline Sheep & 28 & $0 / 28$ \\
\hline Rabbit & 2 & $0 / 2$ \\
\hline Cow & 57 & $13 / 57$ \\
\hline Pig & 64 & $39 / 64$ \\
\hline Goat & 4 & $0 / 4$ \\
\hline Donkey & 4 & $0 / 4$ \\
\hline
\end{tabular}

407

408 Table 6. Comparison of prevalence based on PCR-sequencing between animal 409 species

410

\begin{tabular}{lcccc}
\hline Animal species & $\begin{array}{c}\text { Number of } \\
\text { samples analyzed }\end{array}$ & $\begin{array}{c}\text { Number of } \\
\text { positive samples } \\
\text { by PCR- } \\
\text { Sequencing }\end{array}$ & Prevalence [IC 95\%] & p-value \\
\hline Cat & 105 & 50 & $47.6[37.8-57.6]$ & \\
\hline Dog & 52 & 30 & $57.7[43.2-71.3]$ & \\
\hline Horse & 89 & 24 & $27.0[18.1-37.4]$ & $1.4 \times 10^{-12}$ \\
\hline Sheep & 29 & 28 & $96.6[82.2-99.9]$ & $100.0[15.8-100.0]$ \\
\hline Rabbit & 2 & 2 & 100.0 & \\
\hline
\end{tabular}




\begin{tabular}{lccr}
\hline Cow & 57 & 44 & $77.2[64.2-87.3]$ \\
\hline Pig & 64 & 25 & $39.1[27.1-52.1]$ \\
\hline Goat & 5 & 4 & $80.0[28.4-99.5]$ \\
\hline Donkey & 4 & 4 & $100.0[39.8-100.0]$ \\
\hline
\end{tabular}

411

412 Table 7. Percentage of identity among the sequences obtained.

413

\begin{tabular}{lccc}
\hline \multicolumn{1}{c}{ Animal species } & Percentage $\mathbf{> 9 9 \%}$ & Percentage $\mathbf{< 9 8 . 7 \%}$ & Percentage $<\mathbf{9 5 \%}$ \\
\hline Cat & 50 & 0 & 0 \\
\hline Dog & 30 & 0 & 0 \\
\hline Horse & 7 & 15 & 2 \\
\hline Sheep & 18 & 7 & 3 \\
\hline Rabbit & 2 & 0 & 3 \\
\hline Cow & 29 & 12 & 5 \\
\hline Pig & 16 & 4 & 2 \\
\hline Goat & 0 & 2 & 0 \\
\hline Donkey & 1 & 3 & \\
\hline
\end{tabular}

414

415 Table 8. Summary of the results of multispacer sequence typing.

416

\begin{tabular}{|c|c|c|c|c|c|c|c|}
\hline \multirow[t]{2}{*}{ Samples } & \multirow[t]{2}{*}{ Origin } & \multirow{2}{*}{$\begin{array}{l}\text { Collection } \\
\text { sites }\end{array}$} & \multicolumn{5}{|c|}{ Genotype } \\
\hline & & & Spacer1 & Spacer2 & Spacer 3 & Spacer 4 & Spacer type* \\
\hline 1 & Sheep & Bourganeuf & & & $x$ & & 1 \\
\hline 2 & Horse & Marseille & & & $x$ & & 1 \\
\hline 3 & Pig & Avignon & $x$ & $x$ & $x$ & $x$ & 2 \\
\hline 4 & Pig & Avignon & $x$ & $x$ & $x$ & $x$ & 2 \\
\hline 5 & Pig & Avignon & $x$ & $x$ & $x$ & $x$ & 2 \\
\hline 6 & Pig & Avignon & $x$ & $x$ & $x$ & $x$ & 2 \\
\hline 7 & Pig & Avignon & $x$ & $x$ & $x$ & & 3 \\
\hline 8 & Pig & Avignon & $x$ & $x$ & & & 4 \\
\hline 9 & Pig & Avignon & & $x$ & $x$ & & 5 \\
\hline 10 & Pig & Avignon & & & $x$ & & 1 \\
\hline 11 & Pig & Avignon & $x$ & $x$ & $x$ & $x$ & 2 \\
\hline 12 & Pig & Avignon & $x$ & $x$ & $x$ & & 3 \\
\hline 13 & Pig & Avignon & $x$ & $x$ & $x$ & $x$ & 2 \\
\hline 14 & Pig & Avignon & $x$ & $x$ & $x$ & $x$ & 2 \\
\hline 15 & Dog & Marseille & $x$ & $x$ & $x$ & $x$ & 2 \\
\hline 16 & Dog & Marseille & $x$ & $x$ & $x$ & $x$ & 2 \\
\hline 17 & Dog & Marseille & $x$ & $x$ & $x$ & & 3 \\
\hline 18 & Dog & Marseille & $x$ & $x$ & $x$ & & 3 \\
\hline 19 & Dog & Marseille & $x$ & $x$ & $x$ & $x$ & 2 \\
\hline 20 & Dog & Marseille & & & $x$ & & 1 \\
\hline 21 & Cat & Marseille & & & $x$ & & 1 \\
\hline 22 & Cat & Marseille & & & $x$ & & 1 \\
\hline 23 & Cat & Marseille & & & $x$ & & 1 \\
\hline 24 & Cat & Marseille & & & $X$ & & 1 \\
\hline
\end{tabular}




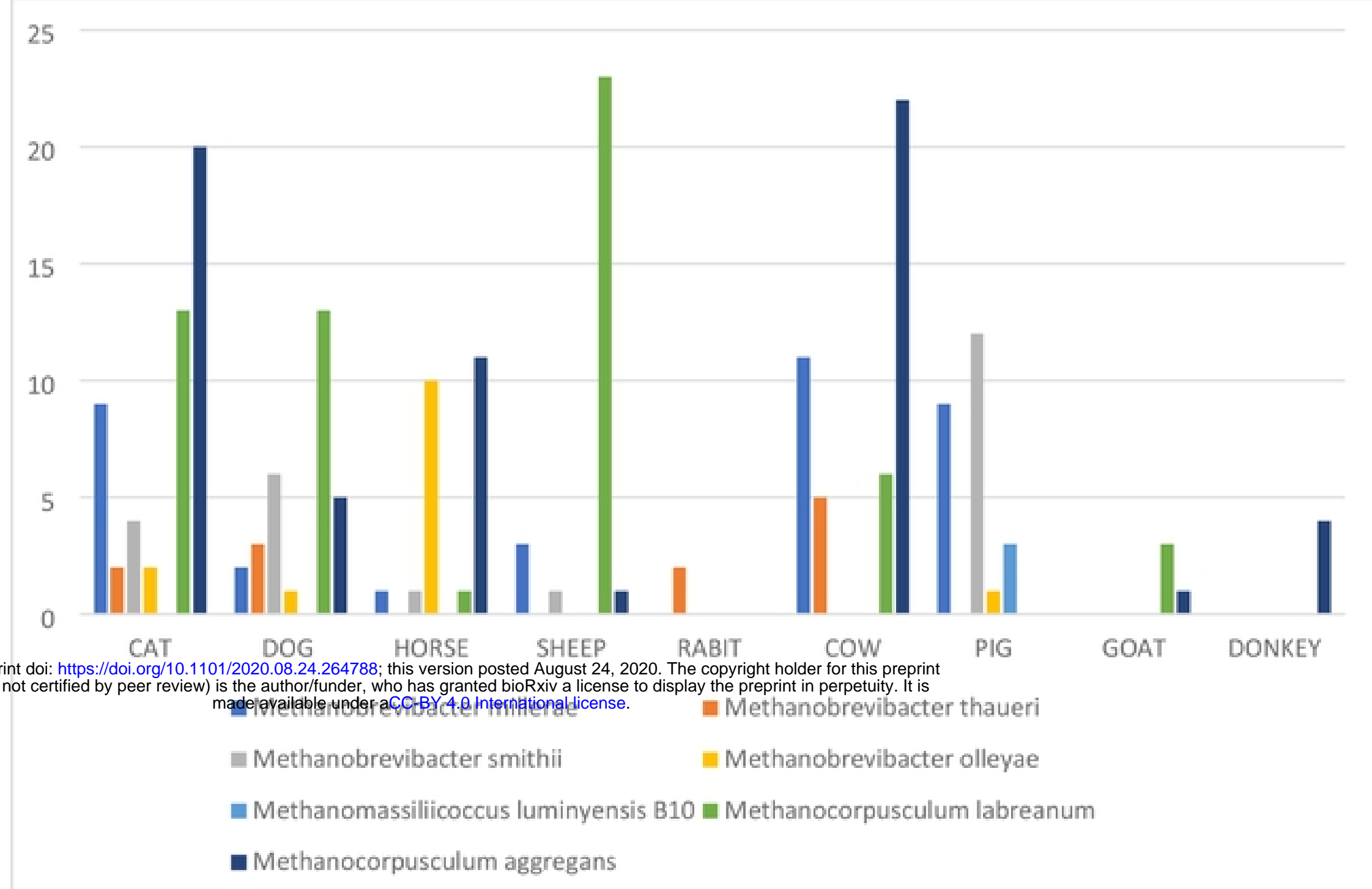

Figure 1. Species detected per sample according to their quantity.

Figure 1 


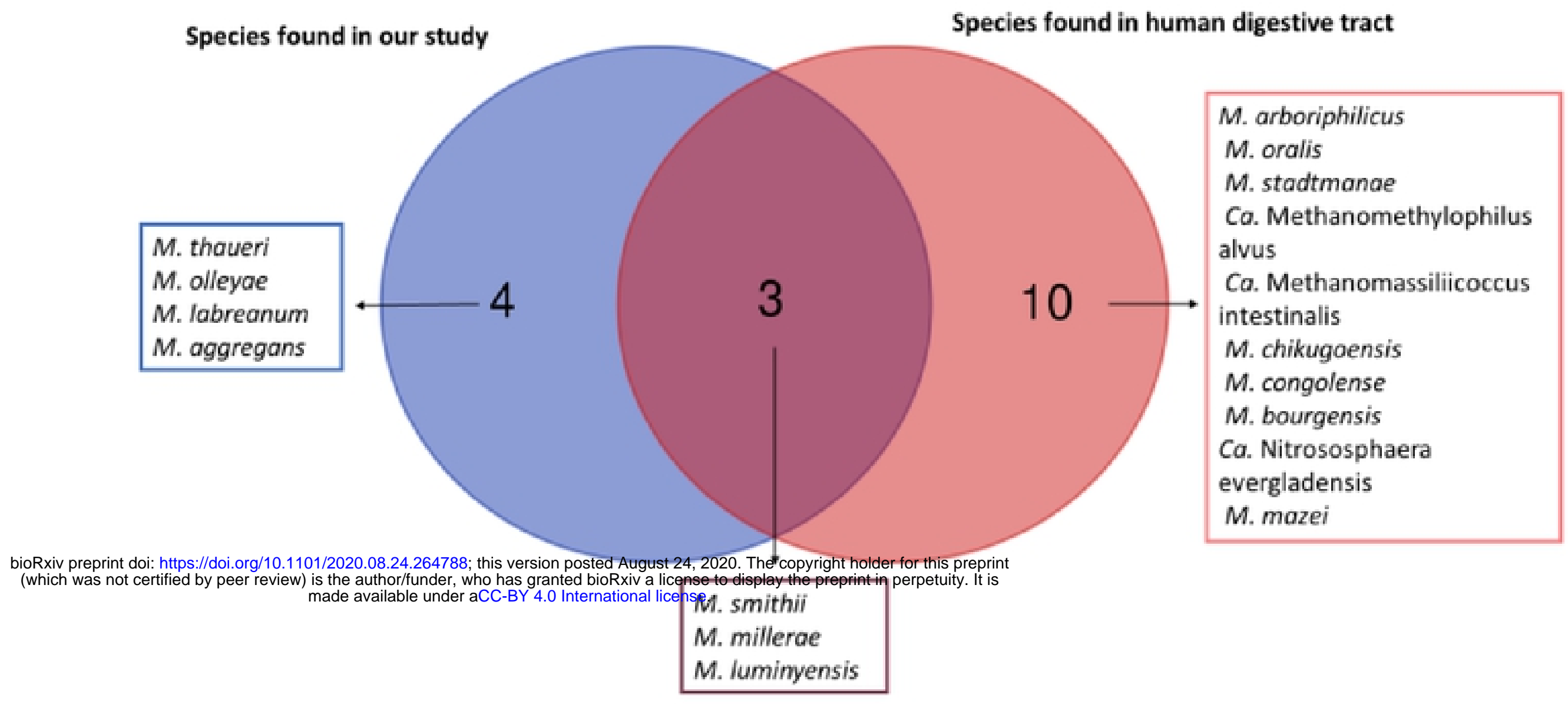

Figure 2. Venn diagram between the methanogens found in our study and those known from the human digestive tract. 


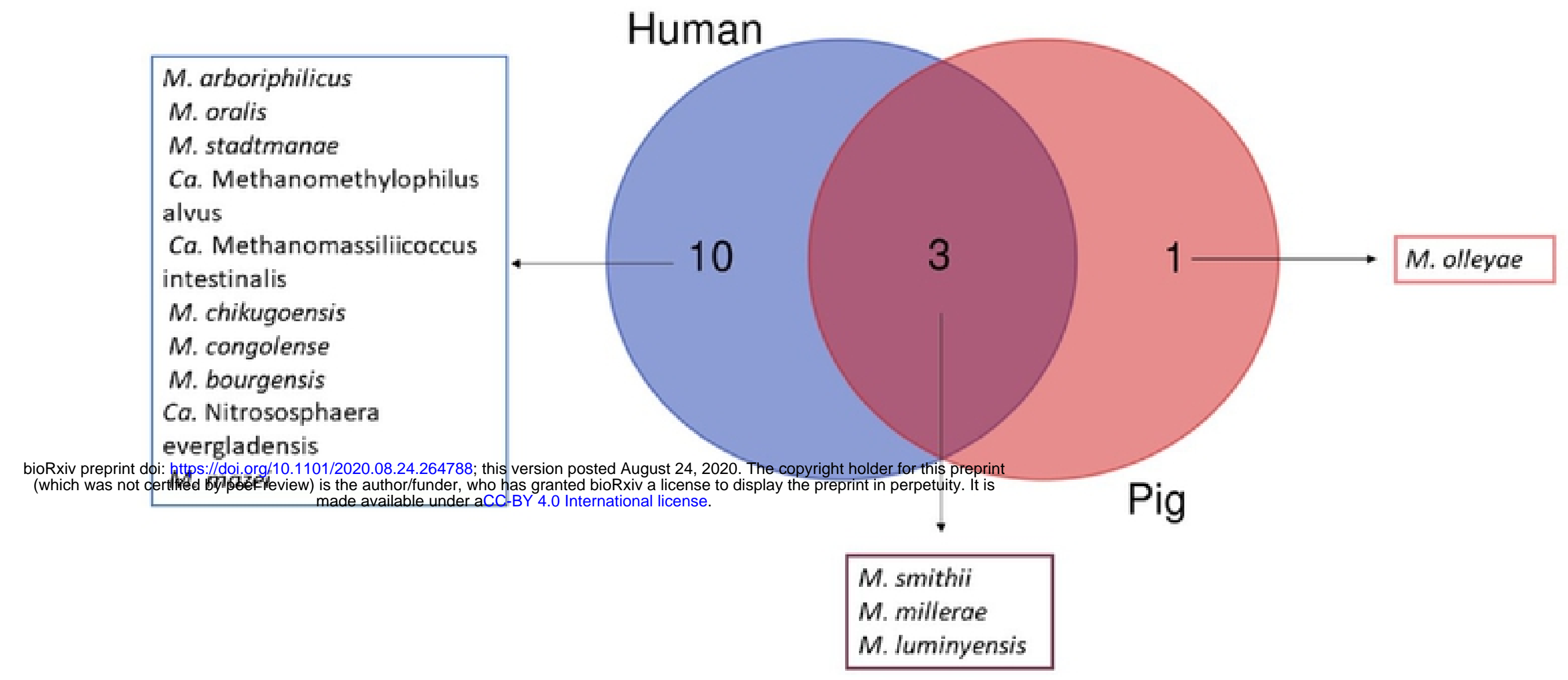

Figure 3. Venn diagram between the methanogens found in pigs and those known from the human digestive tract.

Figure 3 


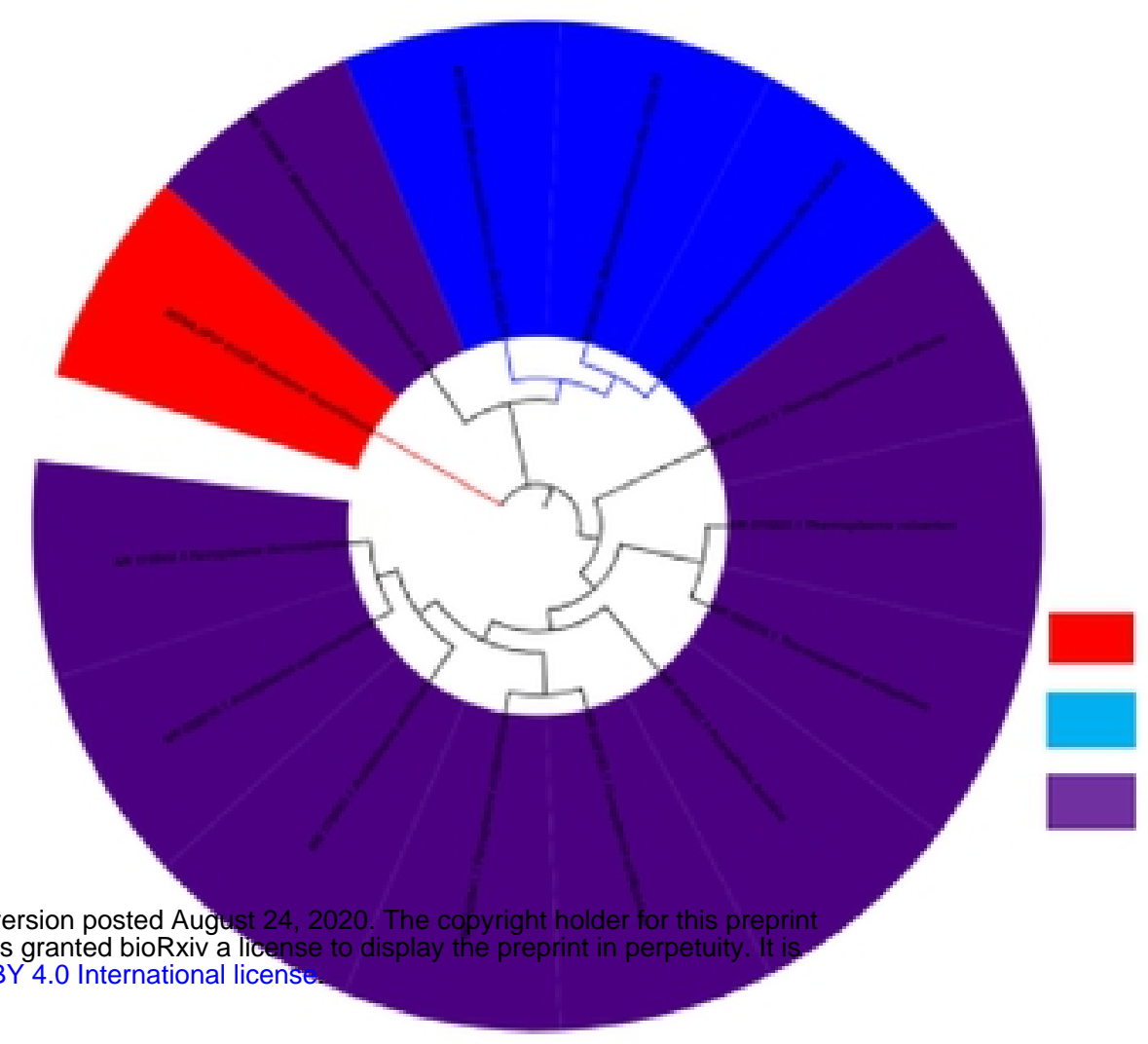

Control out of group

New genera of methanogens

Top Ten Hits

Figure 4. Molecular phylogenetic analysis, based on 16S rRNA partial gene, showed the position of Methanomassiliicoccus-like sequences detected in feces of pig. The evolutionary history was inferred using the Neighbor-Joining method. The optimal tree with the sum of branch length $=0.82722721$ is shown. The percentage of replicate trees in which the associated taxa clustered together in the bootstrap test (1.000 replicates) are shown next to the branches. The tree is drawn to scale, with branch lengths in the same units as those of the evolutionary distances used to infer the phylogenetic tree. The evolutionary distances were computed using the Maximum Composite Likelihood method and are in the units of the number of base substitutions per site. The analysis involved 14 nucleotide sequences. All positions containing gaps and missing data were eliminated. There was a total of 415 positions in the final dataset. Evolutionary analyses were conducted in MEGA7. 


\section{SUPPLEMENTARY FIGURES}

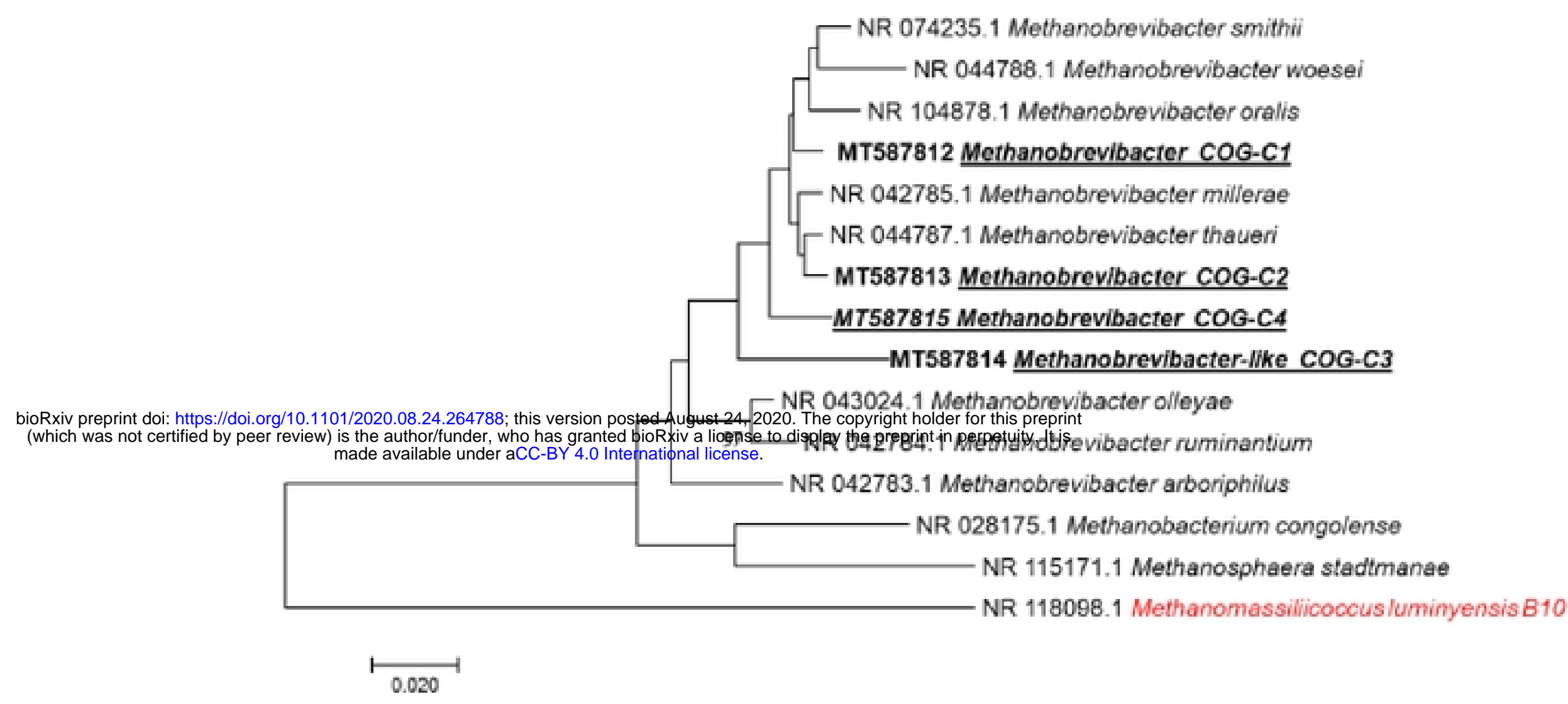

Supplementary Figure 1. Molecular phylogenetic analysis, based on 16S rRNA partial gene, showed the position of Methanobrevibacter sequences detected in feces of cow. The evolutionary history was inferred using the Neighbor-Joining method. The optimal tree with the sum of branch length $=0.56972409$ is shown. The percentage of replicate trees in which the associated taxa clustered together in the bootstrap test (1.000 replicates) are shown next to the branches. The tree is drawn to scale, with branch lengths in the same units as those of the evolutionary distances used to infer the phylogenetic tree. The evolutionary distances were computed using the Maximum Composite Likelihood method and are in the units of the number of base substitutions per site. The analysis involved 15 nucleotide sequences. All positions containing gaps and missing data were eliminated. There was a total of 447 positions in the final dataset. Evolutionary analyses were conducted in MEGA7. Bootstrap values $\geq 95 \%$ are indicated at nodes. 


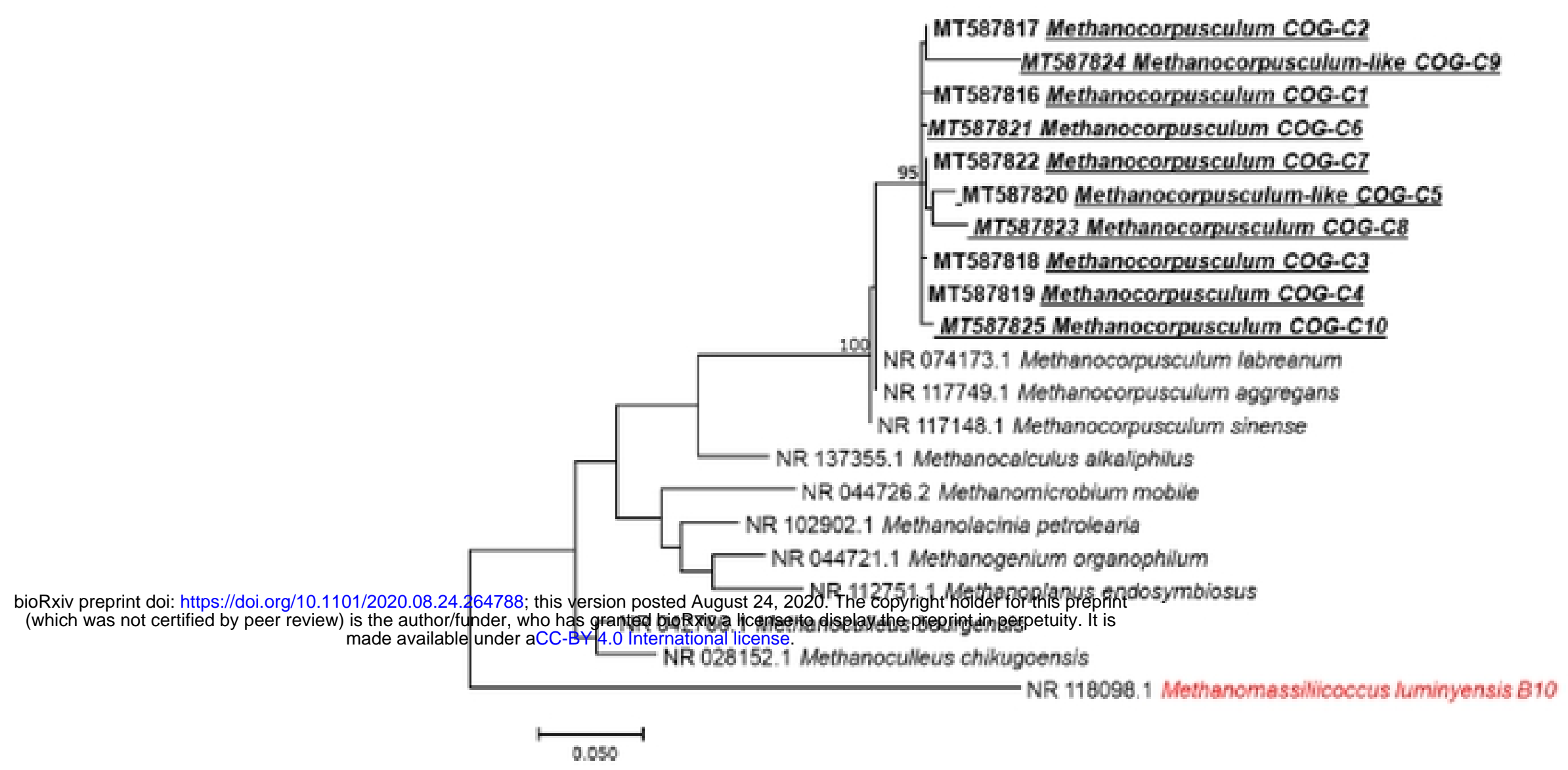

Supplementary Figure 2. Molecular phylogenetic analysis, based on 16S rRNA partial gene, showed the position of Methanocorpusculum sequences detected in feces of cow. The evolutionary history was inferred by using the Maximum Likelihood method based on the Kimura 2-parameter model. The tree with the highest log likelihood (-1869.71) is shown. The percentage of trees in which the associated taxa clustered together is shown next to the branches. Initial tree(s) for the heuristic search were obtained automatically by applying Neighbor-Join and BioNJ algorithms to a matrix of pairwise distances estimated using the Maximum Composite Likelihood (MCL) approach, and then selecting the topology with superior log likelihood value. The tree is drawn to scale, with branch lengths measured in the number of substitutions per site. The analysis involved 21 nucleotide sequences. All positions containing gaps and missing data were eliminated. There was a total of 375 positions in the final dataset. Evolutionary analyses were conducted in MEGA7. Bootstrap values $\geq 95 \%$ are indicated at nodes. 


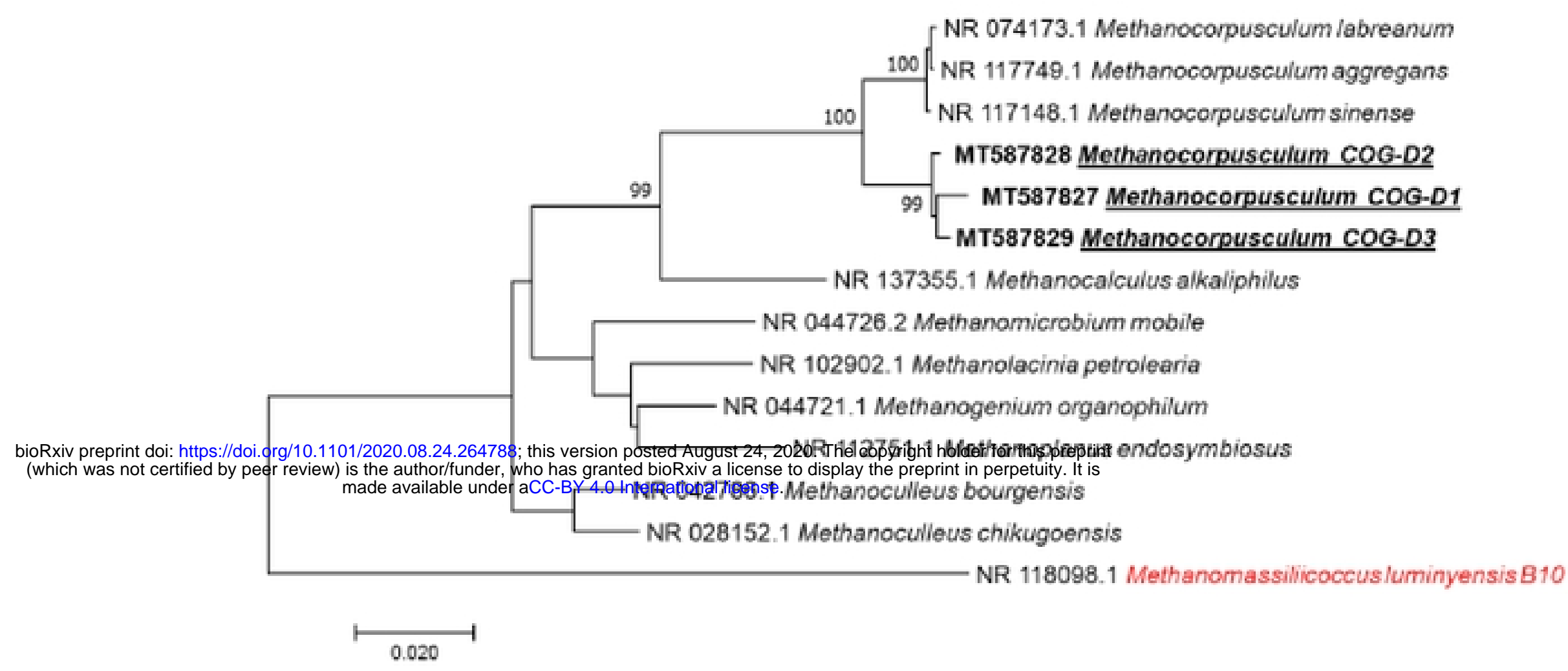

Supplementary Figure 3. Molecular phylogenetic analysis, based on 16S rRNA partial gene, showed the position of Methanocorpusculum sequences detected in feces of donkey. The evolutionary history was inferred using the Neighbor-Joining method. The optimal tree with the sum of branch length $=0.41522849$ is shown. The percentage of replicate trees in which the associated taxa clustered together in the bootstrap test ( 1.000 replicates) are shown next to the branches. The tree is drawn to scale, with branch lengths in the same units as those of the evolutionary distances used to infer the phylogenetic tree. The evolutionary distances were computed using the Maximum Composite Likelihood method and are in the units of the number of base substitutions per site. The analysis involved 14 nucleotide sequences. All positions containing gaps and missing data were eliminated. There was a total of 476 positions in the final dataset. Evolutionary analyses were conducted in MEGA7. Bootstrap values $\geq 95 \%$ are indicated at nodes. 


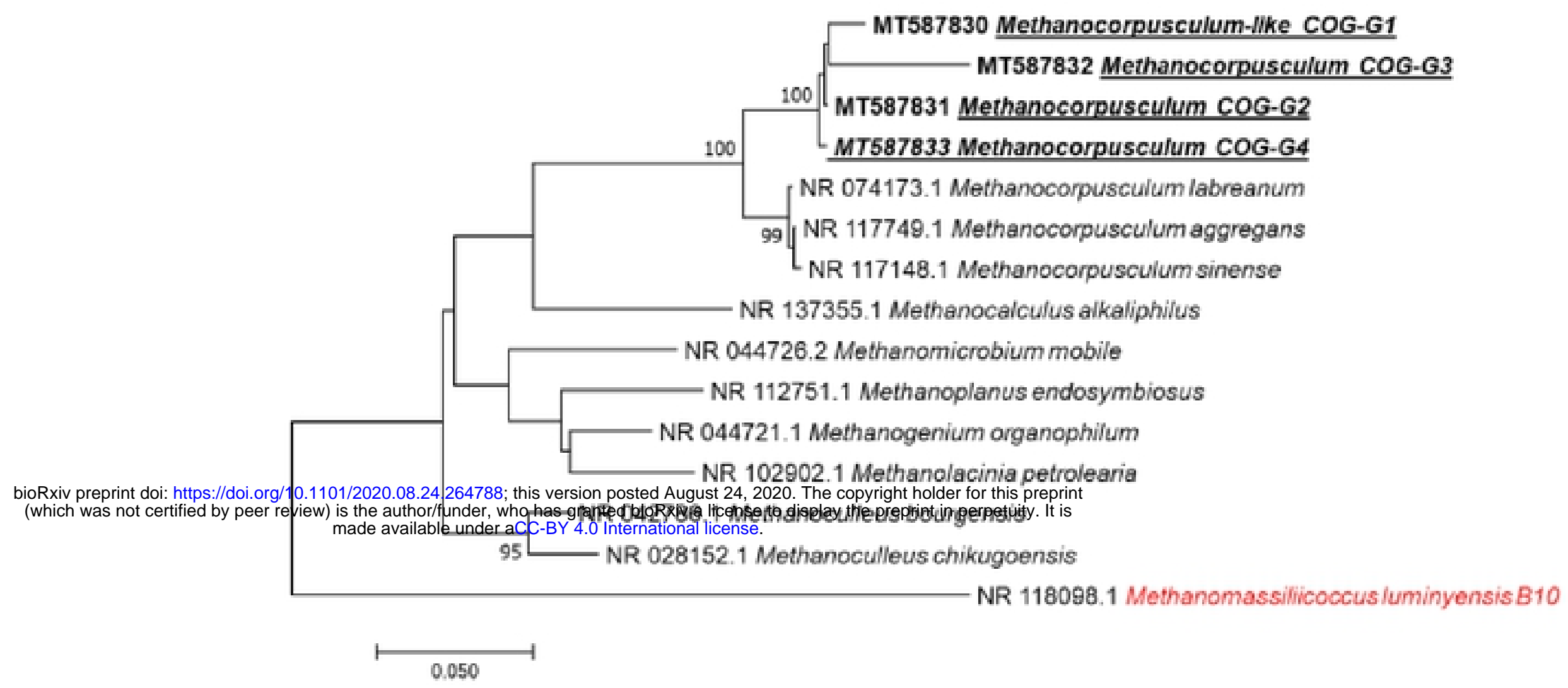

Supplementary Figure 4. Molecular phylogenetic analysis, based on 16S rRNA partial gene, showed the position of Methanocorpusculum sequences detected in feces of goat. The evolutionary history was inferred using the Neighbor-Joining method. The optimal tree with the sum of branch length $=0.79665377$ is shown. The percentage of replicate trees in which the associated taxa clustered together in the bootstrap test ( 1.000 replicates) are shown next to the branches. The tree is drawn to scale, with branch lengths in the same units as those of the evolutionary distances used to infer the phylogenetic tree. The evolutionary distances were computed using the Maximum Composite Likelihood method and are in the units of the number of base substitutions per site. The analysis involved 15 nucleotide sequences. All positions containing gaps and missing data were eliminated. There was a total of 427 positions in the final dataset. Evolutionary analyses were conducted in MEGA7. Bootstrap values $\geq 95 \%$ are indicated at nodes. 


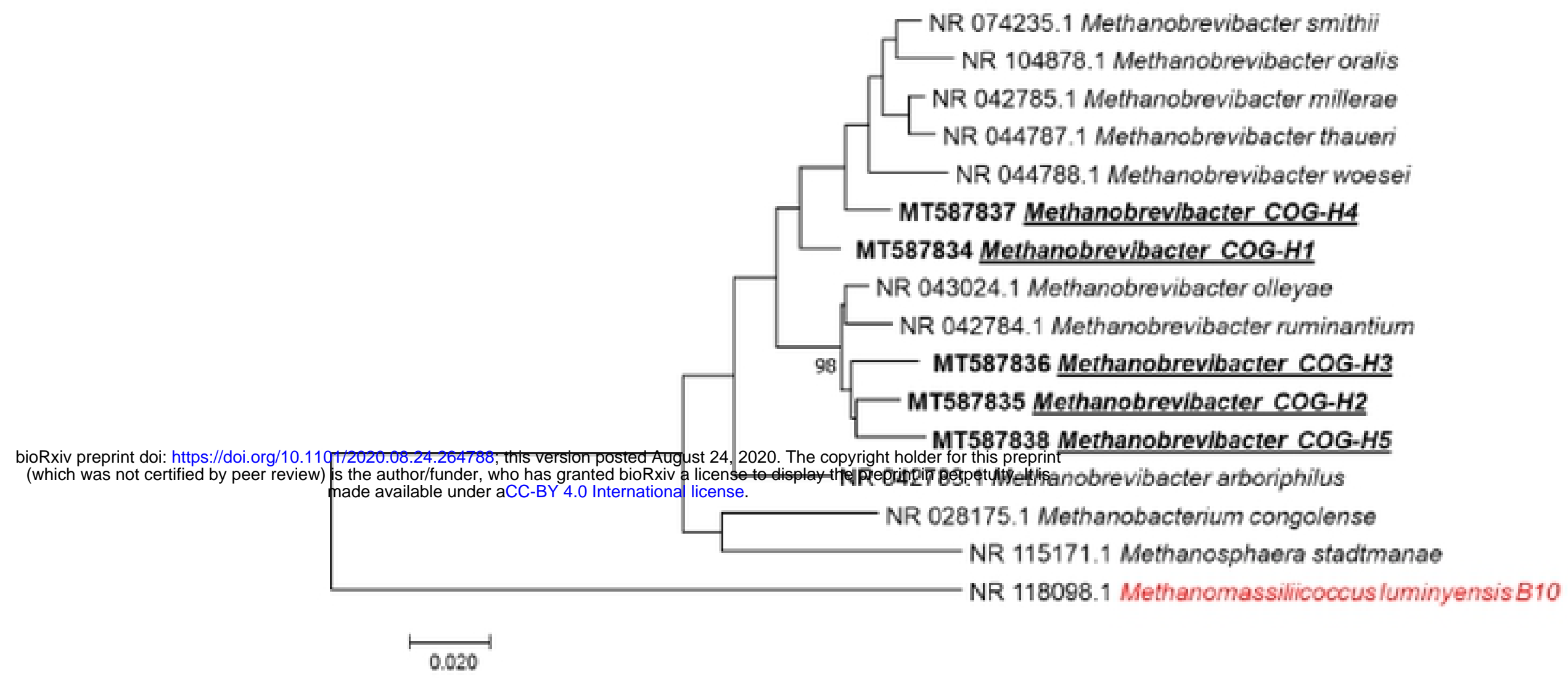

Supplementary Figure 5. Molecular phylogenetic analysis, based on 16S rRNA partial gene, showed the position of Methanobrevibacter sequences detected in feces of horse. The evolutionary history was inferred using the Neighbor-Joining method. The optimal tree with the sum of branch length $=0.58360989$ is shown. The percentage of replicate trees in which the associated taxa clustered together in the bootstrap test ( 1.000 replicates) are shown next to the branches. The tree is drawn to scale, with branch lengths in the same units as those of the evolutionary distances used to infer the phylogenetic tree. The evolutionary distances were computed using the Maximum Composite Likelihood method and are in the units of the number of base substitutions per site. The analysis involved 16 nucleotide sequences. All positions containing gaps and missing data were eliminated. There was a total of 408 positions in the final dataset. Evolutionary analyses were conducted in MEGA7. Bootstrap values $\geq 95 \%$ are indicated at nodes. 


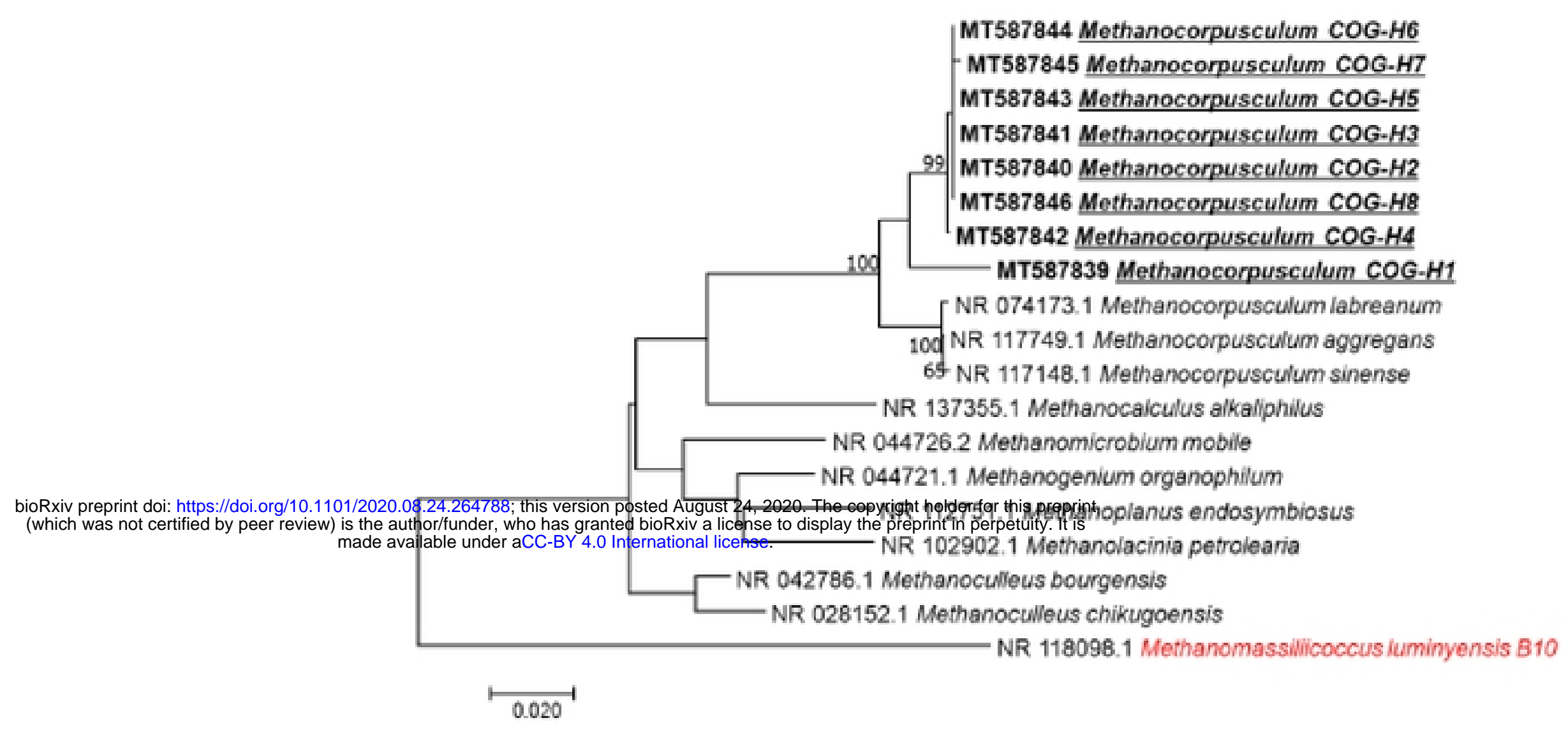

Supplementary Figure 6. Molecular phylogenetic analysis, based on 16S rRNA partial gene, showed the position of Methanocorpusculum sequences detected in feces of horse. The evolutionary history was inferred using the Neighbor-Joining method. The optimal tree with the sum of branch length $=0.52514892$ is shown. The percentage of replicate trees in which the associated taxa clustered together in the bootstrap test ( 1.000 replicates) are shown next to the branches. The tree is drawn to scale, with branch lengths in the same units as those of the evolutionary distances used to infer the phylogenetic tree. The evolutionary distances were computed using the Maximum Composite Likelihood method and are in the units of the number of base substitutions per site. The analysis involved 19 nucleotide sequences. All positions containing gaps and missing data were eliminated. There was a total of 417 positions in the final dataset. Evolutionary analyses were conducted in MEGA7. Bootstrap values $\geq 95 \%$ are indicated at nodes. 


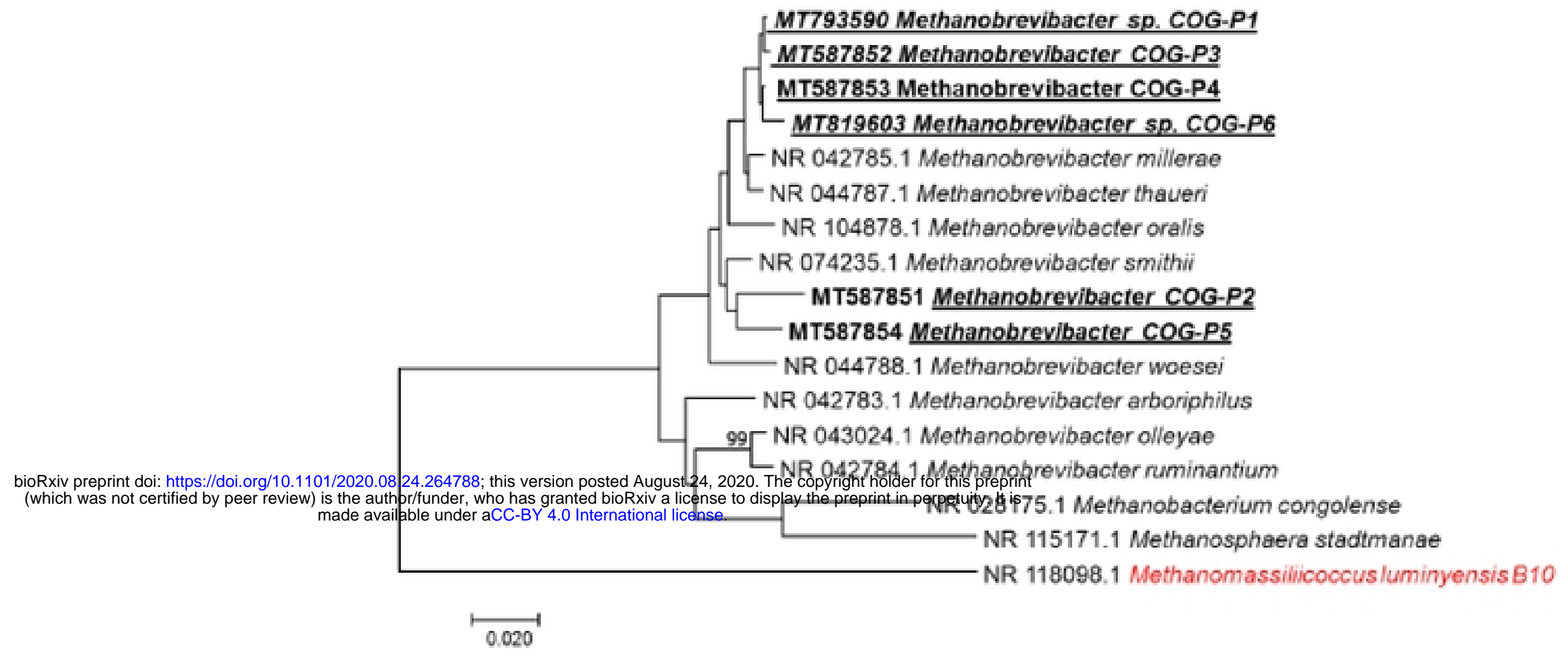

Supplementary Figure 7. Molecular phylogenetic analysis, based on 16S rRNA partial gene, showed the position of Methanobrevibacter sequences detected in feces of pig. The evolutionary history was inferred using the Neighbor-Joining method. The optimal tree with the sum of branch length $=0.56411513$ is shown. The percentage of replicate trees in which the associated taxa clustered together in the bootstrap test ( 1.000 replicates) are shown next to the branches. The tree is drawn to scale, with branch lengths in the same units as those of the evolutionary distances used to infer the phylogenetic tree. The evolutionary distances were computed using the Maximum Composite Likelihood method and are in the units of the number of base substitutions per site. The analysis involved 17 nucleotide sequences. All positions containing gaps and missing data were eliminated. There was a total of 467 positions in the final dataset. Evolutionary analyses were conducted in MEGA7. Bootstrap values $\geq 95 \%$ are indicated at nodes. 


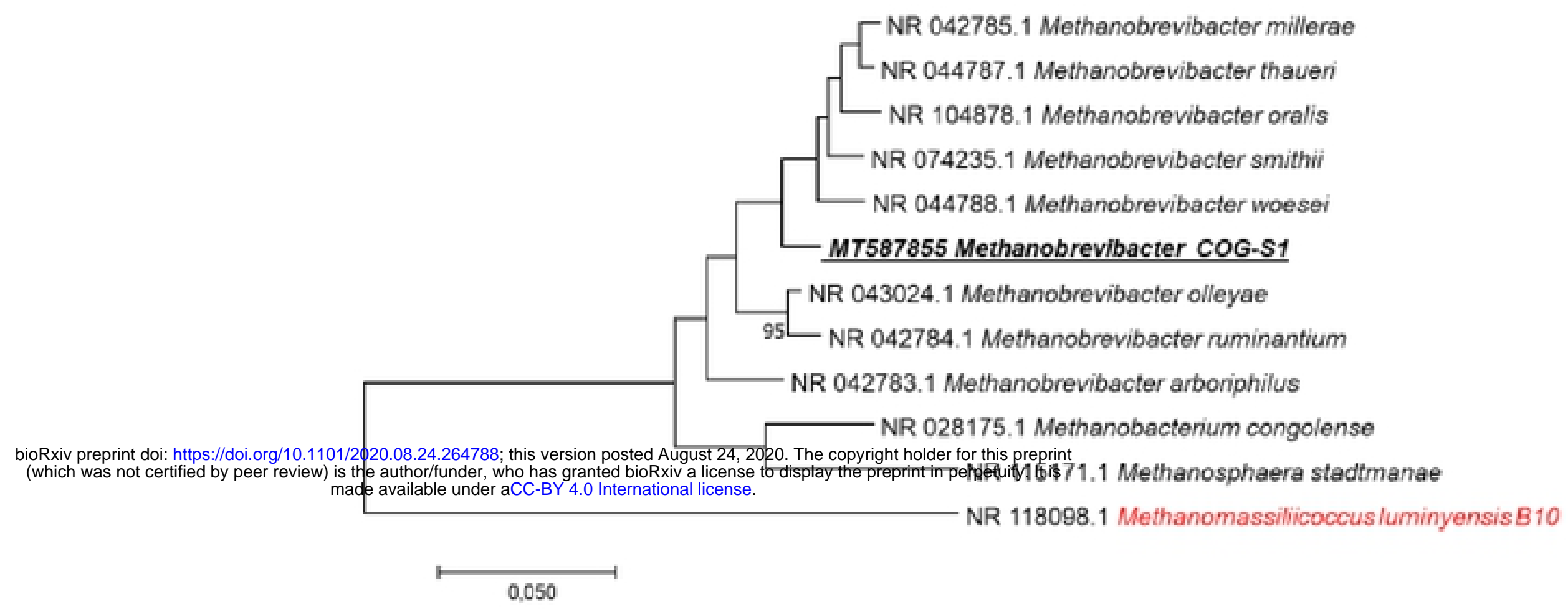

Supplementary Figure 8. Molecular phylogenetic analysis, based on 16S rRNA partial gene, showed the position of Methanobrevibacter sequence detected in feces of sheep. The evolutionary history was inferred by using the Maximum Likelihood method based on the Kimura 2-parameter model. The tree with the highest log likelihood (-1972.37) is shown. The percentage of trees in which the associated taxa clustered together is shown next to the branches. Initial tree(s) for the heuristic search were obtained automatically by applying Neighbor-Join and BioNJ algorithms to a matrix of pairwise distances estimated using the Maximum Composite Likelihood (MCL) approach, and then selecting the topology with superior log likelihood value. The tree is drawn to scale, with branch lengths measured in the number of substitutions per site. The analysis involved 12 nucleotide sequences. All positions containing gaps and missing data were eliminated. There was a total of 554 positions in the final dataset. Evolutionary analyses were conducted in MEGA7. Bootstrap values $\geq 95 \%$ are indicated at nodes. 


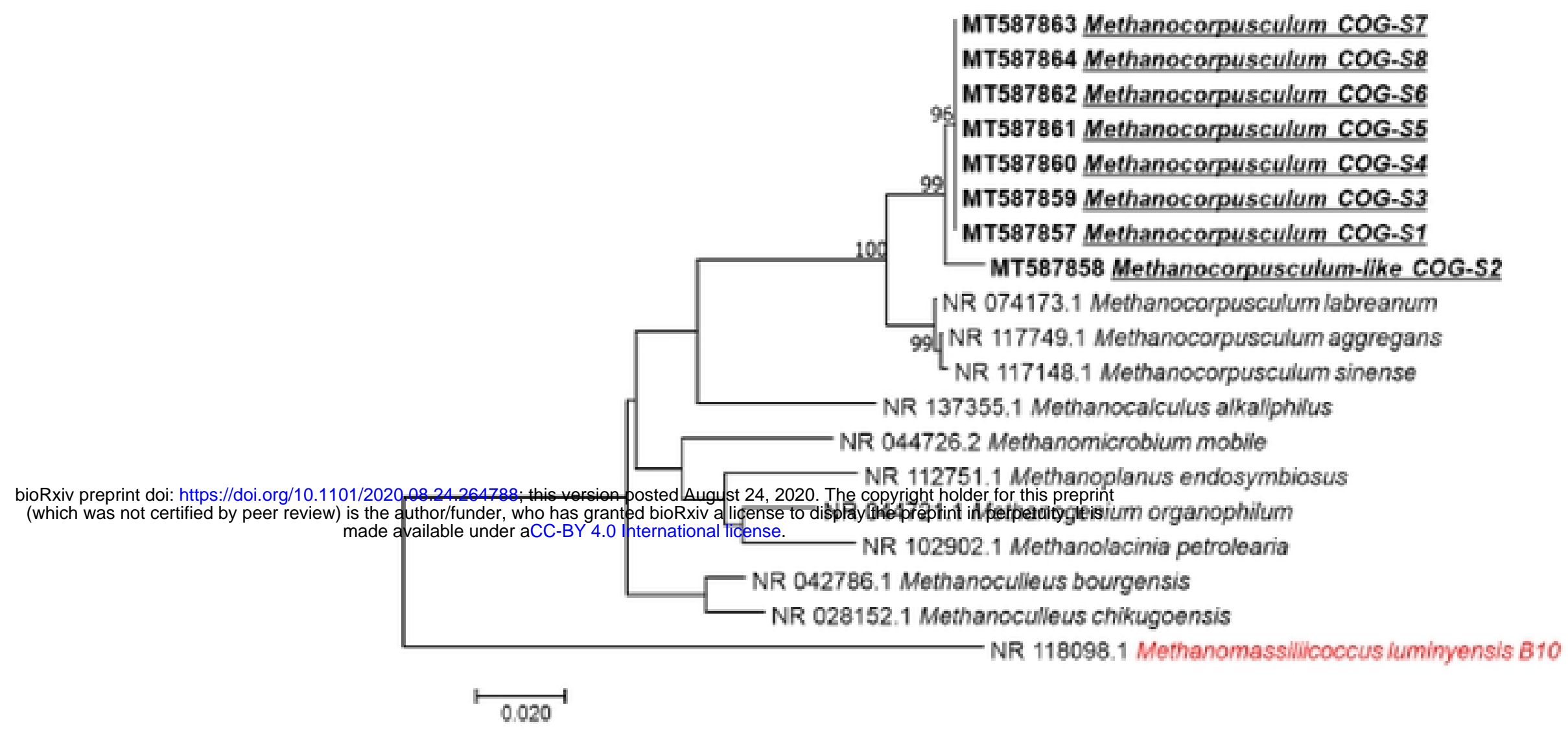

Supplementary Figure 9. Molecular phylogenetic analysis, based on 16S rRNA partial gene, showed the position of Methanocorpusculum sequences detected in feces of sheep. The evolutionary history was inferred using the Neighbor-Joining method. The optimal tree with the sum of branch length $=0.48477167$ is shown. The percentage of replicate trees in which the associated taxa clustered together in the bootstrap test ( 1.000 replicates) are shown next to the branches. The tree is drawn to scale, with branch lengths in the same units as those of the evolutionary distances used to infer the phylogenetic tree. The evolutionary distances were computed using the Maximum Composite Likelihood method and are in the units of the number of base substitutions per site. The analysis involved 19 nucleotide sequences. All positions containing gaps and missing data were eliminated. There was a total of 441 positions in the final dataset. Evolutionary analyses were conducted in MEGA7. Bootstrap values $\geq 95 \%$ are indicated at nodes. 